\title{
Dielectric Coated Slotted Elliptic Antenna Embedded Partly in Infinite Ground Plane
}

\author{
Abdul-Kadir Hamid \\ Department of Electrical and Computer Engineering, University of Sharjah, Sharjah, UAE \\ Corresponding author, E-mail: akhamidesharjah.ac.ae
}

\begin{abstract}
Radiation characteristics of an axially slotted elliptic antenna coated by conventional dielectric or metamaterial material and embedded partly in infinite ground plane are investigated analytically for TM case. The field in each region is written in terms of appropriate radial and angular Mathieu functions with expansion field coefficients. The boundary conditions at the conducting, dielectric coating and ground plane surfaces are enforced exactly to obtain the unknown field expansion coefficients. Results are obtained and plotted for the radiation pattern to show the effect of the presence of the infinite ground plane and parameters of the material dielectric coating.
\end{abstract}

\section{Introduction}

Studies on finding the radiation characteristics analytically by dielectric coated slotted cylindrical antennas are reported by many authors using separation of variables method where the boundary conditions are satisfied exactly at various surfaces [1-6]. The extension of the solution to the radiation by $\mathrm{N}$ slotted elliptical antenna coated with lossy dielectric material was investigated by showing the effect of lossy dielectrics material on its characteristics [7]. Solutions to materials having lossy or lossless metamaterials as well as chiral material gained considerable attention of several researchers [8-10], while solutions having anisotropic materials have been presented by [11-16]. It was shown that the metamaterial coating enhances the gain of the antenna while the anisotropic and chiral materials produce co and cross polarized fields. Embedded circular or elliptical channels as well as circular slotted antennas with dielectric coating in infinite ground plane were analyzed analytically by [17-18].

Embedded slotted elliptic antenna may have application in airplane industries where the ground plane simulates the airplane wings in the presence of the coated elliptic antenna positioned on the body of the plane. Furthermore, the antenna usually requires a supporting ground plane as it cannot be situated in space without support.

To the best of our knowledge, the radiation characteristics from axially slotted elliptic antenna coated by dielectric material and embedded partly in infinite ground plane has not been investigated analytically in the literature as the presence of the infinite ground plane adds two boundary conditions to the solution compared to the one without ground plane. Thus, the tangential electric field should vanish on the external and internal parts of the ground plane. To enforce the tangential field to be zero on the external ground, we express the radiation field in terms of odd Mathieu functions. To enforce the tangential field to be zero on the internal ground field, we need to apply the partial orthogonality property of the angular Mathieu functions.

An exact series solution is presented in this paper to the radiation problem of dielectric coated slotted elliptic antenna embedded partially in ground plane for TM case. The novelty of the reported work is that in using the method of separation of variables to obtain analytic solution to the characteristics of the antenna radiation problem. To do so, fields are expanded in terms of radial and angular Mathieu functions, and their first derivatives, to satisfy the boundary conditions at all surfaces exactly. When the elliptic antenna radiates in the presence of the dielectric coating, the electric and magnetic fields inside the coating will be expressed using radial Mathieu function of the second type as well as angular Mathieu functions.

The exact solution presented in this paper is useful to the electromagnetics community for the following reasons. It can be applied as a benchmark for validating solutions for similar problems derived using numerical or approximate procedures. This solution can be used to show that the method of separation of variables can be employed to solve this type of problems effectively. Furthermore, the analysis presented in this paper, enables one to obtain exact solutions to radiation problems involving objects composed of other of types of materials [19-23].

Section two of the paper presents the formulation of the radiation by slotted elliptic cylinder coated with conventional or metamaterials using Mathieu functions. The boundary conditions are satisfied exactly at various surfaces. The unknown coefficients are obtained using the orthogonally or partial orthogonality property of angular Mathieu functions when the elliptic surface is not complete. Section three presents the far fields and antenna gain as well as the numerical results showing the effect of ground plane on the radiation pattern, gain and conductance of the slotted elliptic antenna while section four contains the conclusions. 


\section{Formulation}

Consider a dielectric coated elliptic antenna with axial slot embedded partly in a ground plane as shown in Fig.1. The parameters $a_{1}$ and $b_{1}$ represent the inner conducting elliptic antenna semi-major and semi-minor axes, respectively, while $a_{2}$ and $b_{2}$ are the semi-major and semi-minor axes of the dielectric coating. The antenna length extends from $v_{1}$ and $v_{2}$ while the ground plane is assumed to be perfectly conducting and situated at the plane $v=0$ and $v=\pi$. The elliptical coordinates $(u, v, z)$ may be expressed in terms of Cartesian coordinate systems $(x, y, z)$ as

$$
\begin{aligned}
& x=F \cosh (u) \cos (v) \\
& y=F \sinh (u) \sin (v)
\end{aligned}
$$

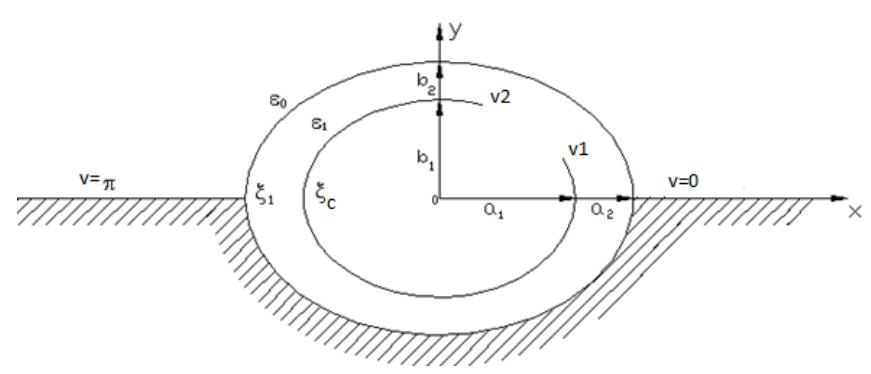

Fig. 1 Slotted elliptic antenna with dielectric coating embedded partly in infinite ground plane.

where $F$ is the elliptical cylinder semi-focal length. The radiated electric field outside region $\xi>\xi_{1}$ in the presence of the ground plane only has an odd form and expressed as

$$
E_{z}^{I}=\sum_{m=1}^{\infty} A_{o m} R_{o m}^{(4)}\left(c_{0}, \xi\right) S_{o m}\left(c_{0}, \eta\right)
$$

where $A_{\text {om }}$ are arbitrary radiated field constants to be determined later by invoking the boundary conditions, $S_{o m}$ are odd angular Mathieu functions of order $m$, while $R_{o m}^{(4)}$ are the odd radial Mathieu functions of the fourth kind, $\xi=\cosh u, \eta=\cos v, c_{0}=k F$, and $k=\omega \sqrt{\mu \varepsilon}$.

Similarly, the electric field within the dielectric coating $\xi_{c}<\xi<\xi_{1}$ will have even and odd solution and expressed as

$$
\begin{aligned}
E_{z}^{I I}= & \sum_{m=0}^{\infty}\left[B_{e m} R_{e m}^{(1)}\left(c_{1}, \xi\right)+C_{e m} R_{e m}^{(2)}\left(c_{1}, \xi\right)\right] S_{e m}\left(c_{1}, \eta\right)+ \\
& \sum_{m=1}^{\infty}\left[B_{o m} R_{o m}^{(1)}\left(c_{1}, \xi\right)+C_{o m} R_{o m}^{(2)}\left(c_{1}, \xi\right)\right] S_{o m}\left(c_{1}, \eta\right)
\end{aligned}
$$

where $c_{1}=k_{1} F, B_{\substack{e m \\ o m}}$ and $C_{\substack{e m \\ o m}}$ are arbitrary constants, $R_{\substack{e m \\ o m}}^{(1)}$ and $R_{\substack{e m \\ o m}}^{(2)}$ are the radial Mathieu functions of the first and second kind and $S_{e m}$ are the even angular Mathieu functions of order $m$. The magnetic field components in regions (I) and (II) are derived using Maxwell's equations and expressed as

$$
\begin{aligned}
H_{v}^{I}= & \frac{-j}{Z_{o} k h} \sum_{m=1}^{\infty} A_{o m} R_{o m}^{(4)}\left(c_{0}, \xi\right) S_{o m}\left(c_{0}, \eta\right) \\
H_{v}^{I I}= & \frac{-j}{Z_{c} k_{1} h}\left\{\sum_{m=0}^{\infty}\left[B_{e m} R_{e m}^{\prime(1)}\left(c_{1}, \xi\right)+C_{e m} R_{e m}^{\prime(2)}\left(c_{1}, \xi\right)\right] S_{e m}\left(c_{1}, \eta\right)\right. \\
& \left.\quad+\sum_{m=1}^{\infty}\left[B_{o m} R_{o m}^{\prime(1)}\left(c_{1}, \xi\right)+C_{o m} R_{o m}^{\prime(2)}\left(c_{1}, \xi\right)\right] S_{o m}\left(c_{1}, \eta\right)\right\}
\end{aligned}
$$

where $h=F \sqrt{\left(\cosh ^{2} u-\cos ^{2} v\right.}, Z_{o}$ is the characteristic wave impedance in free space while $Z_{c}$ is the wave impedance of the dielectric medium. The prime in equations (5) and (6) indicates derivative with respect to coordinate $u$. The unknown field expansion coefficients in equations (3) to (6) are yet to be determined by invoking the boundary conditions.

The boundary conditions at $\xi=\xi_{1}(\pi<\eta<2 \pi)$ require that the tangential electric field component to vanish on the ground plane, and the total tangential electric and magnetic field components to be continuous across the dielectric interface at $\xi=\xi_{1}(0<\eta<\pi)$. Enforcing the boundary condition along with the partial orthogonality property of the angular Mathieu functions, we obtain [17]

$$
\begin{aligned}
& \sum_{m=0}^{\infty}\left[B_{e m} \mathrm{R}_{e m}^{(1)}\left(c_{1}, \xi_{1}\right)+C_{e m} \mathrm{R}_{e m}^{(2)}\left(c_{1}, \xi_{1}\right)\right] Y_{m n}+ \\
& {\left[B_{o n} R_{o n}^{(1)}\left(c_{1}, \xi_{1}\right)+C_{o n} R_{o n}^{(2)}\left(c_{1}, \xi_{1}\right)\right](\pi / 2.0)=0.0}
\end{aligned}
$$

$$
\begin{aligned}
& \sum_{m=1}^{\infty} A_{o m} R_{o m}^{(4)}\left(c_{0}, \xi_{1}\right) Z_{m n}= \\
& \sum_{m=0}^{\infty}\left[B_{e m} \mathrm{R}_{e m}^{(1)}\left(c_{1}, \xi_{1}\right)+C_{e m} \mathrm{R}_{e m}^{(2)}\left(c_{1}, \xi_{1}\right)\right] X_{m n}+ \\
& {\left[B_{o n} R_{o n}^{(1)}\left(c_{1}, \xi_{1}\right)+C_{o n} R_{o n}^{(2)}\left(c_{1}, \xi_{1}\right)\right](\pi / 2.0)}
\end{aligned}
$$

$$
\begin{aligned}
& \frac{Z_{c} k_{1}}{Z_{o} k} \sum_{m=1}^{\infty} A_{o m} R_{o m}^{\prime(4)}\left(c_{0}, \xi_{1}\right) Z_{m n}= \\
& \sum_{m=0}^{\infty}\left[B_{e m} \mathrm{R}_{e m}^{\prime(1)}\left(c_{1}, \xi_{1}\right)+C_{e m} \mathrm{R}_{e m}^{\prime(2)}\left(c_{1}, \xi_{1}\right)\right] X_{m n}+ \\
& {\left[B_{o n} R_{o n}^{\prime(1)}\left(c_{1}, \xi_{1}\right)+C_{o n} R_{o n}^{\prime(2)}\left(c_{1}, \xi_{1}\right)\right](\pi / 2.0)}
\end{aligned}
$$

where 


$$
\begin{aligned}
X_{m n} & =\int_{0}^{\pi} S_{e m}\left(c_{1}, \eta\right) S_{o n}\left(c_{1}, \eta\right) d v \\
Z_{m n} & =\int_{0}^{\pi} S_{o m}\left(c_{1}, \eta\right) S_{o n}\left(c_{0}, \eta\right) d v \\
Y_{m n} & =\int_{\pi}^{2 \pi} S_{e m}\left(c_{1}, \eta\right) S_{o n}\left(c_{1}, \eta\right) d v=-X_{m n}
\end{aligned}
$$

The $\mathrm{z}$ axial component of the electric field on the cylinder surface $\left(\xi=\xi_{c}\right.$ ) should be zero except at the slot location. This leads to

$\sum_{m=0}^{\infty}\left[B_{e m} R_{e m}^{(1)}\left(c_{1}, \xi_{c}\right)+C_{e m} R_{e m}^{(2)}\left(c_{1}, \xi_{c}\right)\right] S_{e m}\left(c_{1}, \eta\right)+$

$\sum_{m=1}^{\infty}\left[B_{o m} R_{o m}^{(1)}\left(c_{1}, \xi_{c}\right)+C_{o m} R_{o m}^{(2)}\left(c_{1}, \xi_{c}\right)\right] S_{o m}\left(c_{1}, \eta\right)=$

$\left\{\begin{array}{cc}f(v) & v_{1}<v<v_{2} \\ 0, & \text { elsewhere. }\end{array}\right.$

Multiplying both sides of (13) by $\underset{\substack{e n \\ o n}}{S_{1}}\left(c_{1}, \eta\right)$ and integrating over $0<v<2 \pi$, we obtain

$$
\begin{aligned}
& {\left[B_{\substack{e n \\
o n}} R_{\substack{e n \\
\text { on }}}^{(1)}\left(c_{1}, \xi_{c}\right)+\underset{\substack{e n \\
o n}}{C_{\substack{e n \\
\text { on }}}} R_{(2)}^{(2)}\left(c_{1}, \xi_{c}\right)\right] N_{\substack{e n \\
o n}}\left(c_{1}\right)=} \\
& f_{e n}=\int_{\substack{v_{1} \\
v_{1}}}^{v_{2}} f(v) S_{\substack{e n \\
o n}}\left(c_{1}, \eta\right) d v
\end{aligned}
$$

where

$$
N_{\substack{e n \\ o n}}\left(c_{1}\right)=\int_{0}^{2 \pi}\left[S_{\substack{e n \\ o n}}\left(c_{1}, \eta\right)\right]^{2} d v
$$

The integrals in equation (14) may be computed numerically by expressing the field at the slot as

$$
\begin{aligned}
& F(v)=E_{0} \cos \left[\pi\left(v-v_{0}\right) /(2 \alpha)\right] \\
& v_{0}=\left(v_{1}+v_{2}\right) / 2 \\
& \alpha=\left(v_{2}-v_{1}\right) / 2
\end{aligned}
$$

where $E_{o}$ is a constant and equation (16) shows that the maximum field is at the center of the slot and vanishes elsewhere. The even and odd angular Mathieu functions are written in terms of Fourier series as

$$
\begin{aligned}
& S_{e n}\left(c_{1}, \eta\right)=\sum_{k} D_{e}^{k}\left(c_{1}, n\right) \cos (k v) \\
& S_{o n}\left(c_{1}, \eta\right)=\sum_{k} D_{o}^{k}\left(c_{1}, n\right) \sin (k v)
\end{aligned}
$$

Substituting equations (16) to (20) into equation (14), $f_{e n}$ and $f_{\text {on }}$ can finally be written in terms of the slot field as

$$
\begin{aligned}
& f_{e n}=E_{o} \sum_{k} D_{e}^{k}\left(c_{1}, n\right) \int_{v_{1}}^{v_{2}} \cos \left[\pi\left(v-v_{0}\right) /(2 \alpha)\right] \cos (k v) d v \\
& f_{o n}=E_{o} \sum_{k} D_{o}^{k}\left(c_{1}, n\right) \int_{v_{1}}^{v_{2}} \cos \left[\pi\left(v-v_{0}\right) /(2 \alpha)\right] \sin (k v) d v
\end{aligned}
$$

The system of equations (7)-(9) and (14) may be written in matrix form as

$$
\left[\begin{array}{lllll}
Q_{11} & Q_{12} & Q_{13} & Q_{14} & Q_{15} \\
Q_{21} & Q_{22} & Q_{23} & Q_{24} & Q_{25} \\
Q_{31} & Q_{32} & Q_{33} & Q_{34} & Q_{35} \\
Q_{41} & Q_{42} & Q_{43} & Q_{44} & Q_{45} \\
Q_{51} & Q_{52} & Q_{53} & Q_{54} & Q_{55}
\end{array}\right]\left[\begin{array}{l}
B_{e} \\
C_{e} \\
B_{o} \\
C_{o} \\
A_{o}
\end{array}\right]=\left[\begin{array}{l}
V_{1} \\
V_{2} \\
V_{3} \\
V_{4} \\
V_{5}
\end{array}\right]
$$

Where the elements of the submatrices are defined in Appendix A. We solve for unknown expansion coefficients from equation (23) by using matrix inversion technique.

\section{Numerical Results}

As After finding the radiated field coefficients $A_{o n}$ from the previous section, the far-field radiation pattern and the antenna gain may be evaluated. The slotted antenna far field for TM case can be written as

$$
E_{z}^{I}(\rho, \varphi)=\sqrt{\frac{j}{k \rho}} e^{-j k \rho}\left[\sum_{n=1}^{\infty} j^{n} A_{o n} S_{o n}\left(c_{0}, \cos \varphi\right)\right]
$$

where $\rho$ and $\varphi$ represent the polar coordinates in the cylindrical coordinate system. The antenna gain for TM case may be expressed as [5]

$$
G(\varphi)=\frac{1}{Z_{o} k \rho S_{a v T M}}\left[\left|\sum_{n=1}^{\infty} j^{n} A_{o n} S_{o n}\left(c_{0}, \cos \varphi\right)\right|^{2}\right]
$$

The aperture conductance per unit length of the slot antenna is defined as [15-16]

$$
G_{a}=2 \pi \rho \frac{S_{a v T M}}{\left|E_{0}\right|^{2}}
$$

where $S_{a v T M}$ is the average power density averaged over an imaginary cylinder of radius $\rho$ and given as

$$
\begin{aligned}
& S_{\text {avTM }}=\frac{1}{2 \pi Z_{0} k \rho}\left[\sum_{n=1}^{\infty}\left|A_{o n}\right|^{2} N_{o n}\left(c_{0}\right)\right] \\
& N_{\substack{e n \\
o n}}\left(c_{0}\right)=\int_{0}^{2 \pi}\left[S_{\substack{e n \\
o n}}\left(c_{0}, \eta\right)\right]^{2} d v
\end{aligned}
$$

The geometrical parameters of the slotted elliptic antenna are assumed to be $a_{1}=\lambda, b_{1}=\lambda / 2, b_{2}=b_{1}+t$, where $t$ is the 
coating thickness, $v_{0}=90^{\circ}$ and $\alpha=2.8657^{\circ}$ to compare the results with previously published data for slotted elliptic antenna [5-7]. Figure 2 shows the radiation pattern numerical results versus the radiation angle $\varphi$ obtained for dielectric coated slotted antenna values $\left(\varepsilon_{r}=4, \mu_{r}=1\right)$ represented by solid line without the presence of the ground plane, for comparison [5-7], and dotted line with the presence of infinite ground plane.

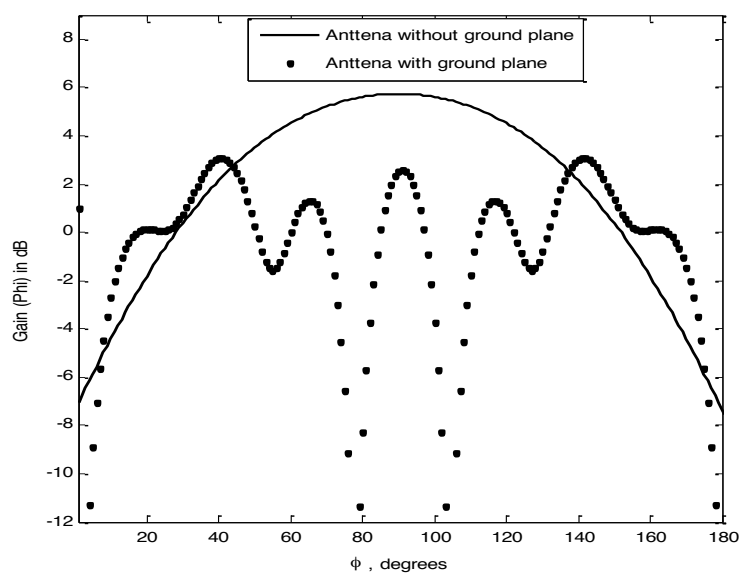

Fig. 2 Radiation pattern versus phi of an axially slotted elliptic cylinder coated with conventional dielectric material. $a_{1}=\lambda \quad, \quad b_{1}=\lambda / 2, \quad b_{2}=b_{1}+0.25 \lambda, \quad v_{0}=90^{\circ} \quad$ and $\alpha=2.8657^{\circ}, \varepsilon_{r}=4.0$ and $\mu_{r}=1.0$.

It can be seen that the effect of the ground plane that reduces the gain at the radiation angle $\varphi=90^{\circ}$ while it increases the gain for radiation angle $\varphi$ less than $40^{\circ}$ and greater than $140^{\circ}$. The field also vanishes, as expected, at the location of the ground plane $\varphi=0^{\circ}$ and $90^{\circ}$.

The effect of the antenna coating thickness is illustrated in Fig. 3 for the same geometrical parameters as in Fig. 2. It can be seen by increase the thickness of the coating it enhances the gain at $\mathrm{t}=0.2 \lambda$ while it decreases the gain at $t$ $\begin{array}{llll}=0.3 & \text { and } & \varphi & =90^{\circ}\end{array}$

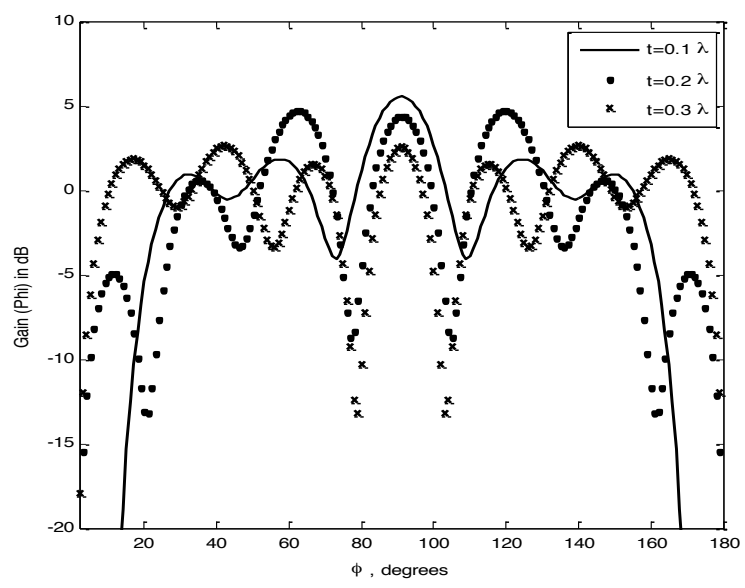

Fig. 3 Radiation pattern versus phi of an axially slotted elliptic cylinder coated by conventional material and partly embedded in ground plane. $a_{1}=\lambda, b_{1}=\lambda / 2, v_{0}=90^{\circ}$, $\alpha=2.8657^{\circ}, \varepsilon_{r}=3.0$ and $\mu_{r}=1.0$.

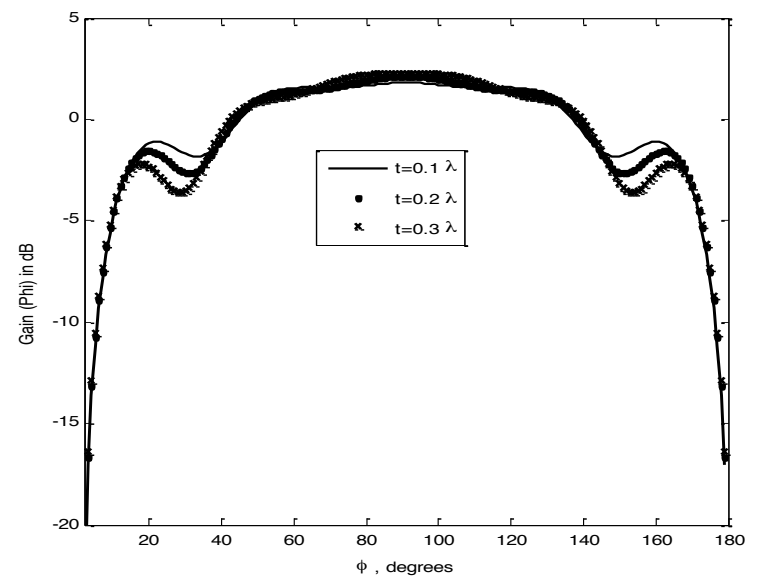

Fig. 4 Radiation pattern versus phi of an axially slotted elliptic cylinder coated by metamaterial and partly embedded in ground plane. $a_{1}=\lambda, b_{1}=\lambda / 2, v_{0}=90^{\circ}$, $\alpha=2.8657^{\circ}, \varepsilon_{r}=-3.0$ and $\mu_{r}=1.0$.

Fig. 4 has the same geometrical parameters as in Fig. 3 except the antenna is coated with metamaterial $\varepsilon_{r}=-3.0$. The thickness of the antenna has no effect on the gain, in case, especially on the location of the antenna. The gain versus coating thickness for a slotted elliptic antenna with the same geometrical parameters used in Fig. 2 is plotted in Fig. 5. The gain is evaluated at $\varphi=90^{\circ}$ since the slot is centered at $v=90^{\circ}$ where the gain is expected to be maximum. It can be seen that the gain increases with thickness.

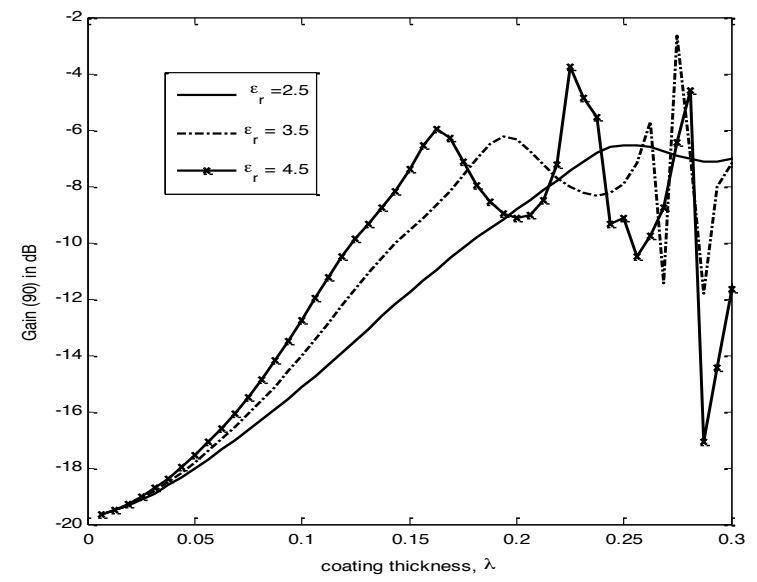

Fig. 5 Gain versus coating thickness for an axially slotted elliptic cylinder partly embedded in ground plane. $a_{1}=\lambda$, $b_{1}=\lambda / 2, b_{2}=b_{1}+t, v_{0}=90^{\circ}, \alpha=2.8657^{\circ}, \mu_{r}=1.0$. 


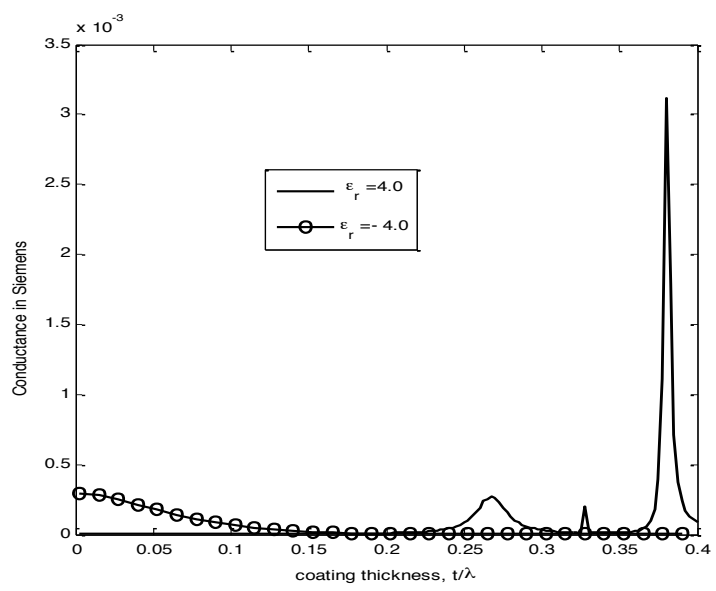

Fig. 6 Aperture conductance versus coating thickness for an axially slotted elliptic cylinder coated by metamaterial and partly embedded in ground plane. $a_{1}=\lambda / 2, b_{1}=\lambda / 4$, $v_{0}=90^{\circ}, \alpha=2.8657^{\circ}$, and $\mu_{r}=1.0$.

The aperture conductance for conventional dielectric or metamaterial coating is shown in Fig. 6 for an elliptical antenna with $a_{1}=\lambda / 2, b_{1}=\lambda / 4, v_{0}=90^{\circ}, \alpha=2.8657^{\circ}$, and $\mu_{r}=1.0$.

\section{Conclusions}

The radiation characteristics of an axially slotted elliptic antenna coated by conventional or dielectric metamaterial and in the presence of infinite ground plane were investigated analytically using the boundary value method. The fields were expressed in terms of appropriated Mathieu functions in every region and the boundary conditions were satisfied exactly at the various surfaces. The resulting system of equations was written in matrix form and the unknown radiation expansion coefficients were obtained by solving the system of equations by matrix inversion. The computed results were compared with the published analytical solution for the case of slotted elliptical antenna coated with conventional dielectric or metamaterial. It was shown that the presence of infinite ground plane led to reducing the gain at the location of the antenna in the case of conventional dielectric coating while the gain was not affected when the antenna was coated with dielectric metamaterial for the presented numerical cases.

\section{Acknowledgments}

The author would like to acknowledge the support by the University of Sharjah, United Arab Emirates.

\section{Appendix A}

$Q_{11}=\mathrm{R}_{e n}^{(1)}\left(c_{1}, \xi_{c}\right) N_{e n}\left(c_{1}\right)$

$$
\begin{aligned}
& Q_{12}=\mathrm{R}_{e n}^{(2)}\left(c_{1}, \xi_{c}\right) N_{e n}\left(c_{1}\right) \\
& Q_{13}=Q_{14}=Q_{15}=0 \\
& Q_{12}=Q_{22}=Q_{25}=0 \\
& Q_{23}=\mathrm{R}_{o n}^{(1)}\left(c_{1}, \xi_{c}\right) N_{o n}\left(c_{1}\right) \\
& Q_{24}=\mathrm{R}_{o n}^{(2)}\left(c_{1}, \xi_{c}\right) N_{o n}\left(c_{1}\right) \\
& Q_{31}=\mathrm{R}_{e m}^{(1)}\left(c_{1}, \xi_{1}\right) Y_{m n} \\
& Q_{32}=\mathrm{R}_{e m}^{(2)}\left(c_{1}, \xi_{1}\right) Y_{m n} \\
& Q_{33}=\mathrm{R}_{e n}^{(1)}\left(c_{1}, \xi_{1}\right)(\pi / 2) \\
& Q_{34}=\mathrm{R}_{e n}^{(2)}\left(c_{1}, \xi_{1}\right)(\pi / 2) \\
& Q_{35}=0 \\
& Q_{41}=\mathrm{R}_{e m}^{(1)}\left(c_{1}, \xi_{1}\right) X_{m n} \\
& Q_{42}=\mathrm{R}_{e m}^{(2)}\left(c_{1}, \xi_{1}\right) X_{m n} \\
& Q_{43}=\mathrm{R}_{o n}^{(1)}\left(c_{1}, \xi_{1}\right)(\pi / 2) \\
& Q_{44}=\mathrm{R}_{o n}^{(2)}\left(c_{1}, \xi_{1}\right)(\pi / 2) \\
& Q_{45}=-R_{o m}^{(4)}\left(c_{0}, \xi_{1}\right) Z_{m n} \\
& Q_{51}=\mathrm{R}_{e m}^{\prime(1)}\left(c_{1}, \xi_{1}\right) X_{m n} \\
& Q_{52}=\mathrm{R}_{e m}^{\prime(2)}\left(c_{1}, \xi_{1}\right) X_{m n} \\
& Q_{53}=\mathrm{R}_{o n}^{(1)}\left(c_{1}, \xi_{1}\right)(\pi / 2) \\
& Q_{54}=\mathrm{R}_{o n}^{(2)}\left(c_{1}, \xi_{1}\right)(\pi / 2) \\
& Q_{55}=-R_{o m}^{(4)}\left(c_{0}, \xi_{1}\right) Z_{m n} \\
& V_{1}=f_{e n}, V_{2}=f_{e n}, V_{3}=V_{4}=V_{5}=0 \\
& A_{o}=B_{o m}, C_{e}=C_{e m}, C_{o}=C_{o m}
\end{aligned}
$$

\section{References}

[1] KNOP, C. M., "External admittance of an axial slot on a dielectric coated metal cylinder," Radio Sci., vol. 3, Aug. 1968 , pp. $803-818$. DOI: $10.1002 /$ rds 196838803

[2] SHAFAI, L., "Radiation from an axial slot antenna coated with a homogenous material," Canadian J. Phys., vol. 50, no. 23, p. 1972. DOI: $10.1139 / \mathrm{p} 72-400$

[3] WONG, J. Y., "Radiation pattern of slotted elliptic cylinder antenna," IEEE Trans. Antennas Propagate., vol. AP-3, Oct. 1955, pp. 200-203. DOI: 10.1109/TAP.1955.1144324

[4] WONG, J. Y., "Radiation conductance of axial and transverse slots in cylinders of elliptical cross section," Proc. IRE, vol. 41, Sept., 1953, pp. 1172-1177. DOI: 10.1109/JRPROC.1953.274453

[5] RICHMOND, J. H., "Axial slot antenna on dielectric coated elliptic cylinder,” IEEE Trans. Antennas Propagate., vol. AP-37, Oct. 1989, pp. 1235-1241. DOI: 10.1109/8.43531

[6] RAGHEB, H. A., SEBAK, A., SHAFAI, L., "Radiation by axial slots on dielectric coated nonconfocal conducting elliptic cylinder," IEE Proc. Microw. Antennas Propagate, vol. 143, no. 2, 1996, pp. 124-130. DOI: 10.1049/ip-map:19960246 
[7] HUSSEIN, M., HAMID, A-K. "Radiation characteristics of $\mathrm{N}$ axial slots on a conducting elliptical antenna coated by a lossy dielectric layer," Canadian Journal of Physics, vol. 82, no. 2, pp. 141-149, 2004. DOI: 10.2528/PIER04082301

[8] KHATIR, B.N., Al-KANHAL, M., SEBAK, "Electromagnetic wave scattering by elliptic chiral cylinder," Journal of Electro-magnetic Waves and Applications, Vol. 20, No. $10, \quad 2006, \quad$ pp. 1377-1390. DOI: $10.1163 / 156939306779276866$

[9] KHATIR, B.N, SEBAK, A., "Slot Antenna on a Conducting Elliptic Cylinder Coated by Chiral Media," Electromagnetics, Vol 29, 2009, pp. 522 - 540. DOI: 10.1080/02726340903167038

[10] KHATIR, B. N., SEBAK, A., "Slot antenna on a conducting elliptic cylinder coated by nonconfocal chiral media, Progress in Electromagnet Research Journal, PIER 93, 2009, PP. 125-143. DOI: 10.2528/PIER09033003

[11] WU, Z.-S, MAO, S.-S., YANGa, L., "Two-dimensional scattering by a conducting elliptic cylinder coated with a homogeneous anisotropic shell," IEEE Trans. Antennas Propagate, vol. 57, 2009, pp. 3638 - 3645. DOI: 10.1109/TAP.2009.2024016

[12] MONTASERI, N., SOLEIMANI, M., ABDOLALI, A., "Realization of the perfect electromagnetic conductor circular cylinder using anisotropic media," Prog. Electromagnet. Res. M, vol. 25, 2012, pp. 173 - 184. DOI: 10.2528/PIERM12062005

[13] WU, X.-B., REN, W., "Axial slot antenna on an anisotropic dielectric-coated circular cylinder," IEE Proceedings Microwaves, Antennas and Propagation, vol. 141, Issue 6, 1994, pp. 527 - 530. DOI: 10.1049/ip-map:19941417

[14] HAMID, A-K., COORAY, F., "Scattering of a plane wave by a homogeneous anisotropic elliptic cylinder", IEEE Transactions on Antennas and Propagation, vol. 63, 8, 2015, pp. 3579 - 3587.

[15] HAMID, A-K., "Axially slotted antenna on elliptic cylinder coated with biaxial anisotropic material," 9th European Conference on Antennas and Propagation (EuCAP 2015), Lisbon, Portugal, April 12-17, 2015. DOI: 10.1109/TAP.2015.2436442

[16] HAMID, A-K., "Series solution to the radiation from slotted antenna on elliptic cylinder coated by biaxial anisotropic material", Applied Computational Electromagnetics Society Journal, vol. 30, 12, 2015, pp. 1301-1305.

[17] HAMID, A-K., "Electromagnetic scattering from a dielectric coated elliptic cylinder loading a semi-elliptic channel in a ground plane," J. of Electromagnetic Waves and Applications, vol. 19, no.2, 2005, pp. 257-269. DOI: $10.1163 / 1569393054497302$

[18] RAGHEB, H., JOHER, U., "Radiation Characteristics of an infinite Dielectric-Coated Axially Slotted Cylindrical Antenna Partly Embedded in a ground plane", IEEE tarns. Antenna and Prog., Vol. 46, no 10, 1998, 1542-1577. DOI: 10.1109/8.725287

[19] HAMID, A-K., "Scattering from an arbitrarily incident plane wave by a PEMC elliptic cylinder confocally coated with a chiral material", Applied Computational Electromagnetics Society Journal, vol. 30, 5, 2015, DOI: 503-509

[20] HAMID, A-K., "Elliptic slotted antenna with anisotropic plasma dielectric coating", International Journal of Advanced Research in Computer and Communication Engineering, vol. 4, no.2, 2015, pp. 1-4. DOI: 0.17148/IJARCCE.2015.4201

[21] HAMID, A-K., "Metamaterial coated slotted elliptic antenna embedded partially in a ground plane", 16th Mediterranean
Microwave Symposium (MMS2016)", Al Ain University of Science and Technology (AAU), Abu Dhabi City - United Arab Emirates (UAE), November 14th-16th, 2016. DOI: 10.1109/MMS.2016.7803804

[22] HAMID, A-K., Cooray, F., "Two-dimensional scattering by a homogeneous Gyrotropic-type elliptic cylinder", Advanced Electromagnetics Journal, vol. 5, 2016, pp. 106-112, 2016. DOI: 10.7716/aem.v5i3.401

[23] RAGHEB, H., HASSAN, E., "Radiation from axial slot on an infinitely long conducting circular cylinder eccentrically coated with dielectric and partly embedded in a ground plane", Progress In Electromagnetics Research, vol. 32, 2013, pp. 271-2013. DOI: 10.2528/PIERM13051604 